\title{
Defining the Molecular Mechanisms for Tubal Ectopic Pregnancy Using Mouse Models
}

\section{Ruijin Shao*}

Department of Physiology/Endocrinology, Institute of Neuroscience and Physiology, The Sahlgrenska Academy at University of Gothenburg, Gothenburg 40530, Sweden

Ectopic pregnancy (EP), defined as extra uterine pregnancy due to embryo implantation outside the uterus, is a major cause of human maternal morbidity and mortality [1,2]. It accounts for $1.5-2 \%$ of all pregnancies in the Western world [3], and more than $98 \%$ of ectopic pregnancies are in the Fallopian tube [2]. Tubal EP is a growing problem in developing countries and increases annual health care costs $[3,4]$. The pathophysiology of EPs is poorly understood, it is therefore difficult to predict the development of EP, and treatment and prevention options are limited [5].

The mammalian Fallopian tube is a dynamic, steroid-responsive tissue [6] composed of heterogeneous cell types: ciliated and secretory epithelial cells as well as smooth muscle cells, all of which appear to be specialised to perform different functions $[7,8]$. The tubal epithelium is normally not receptive to implantation and acts as a mechanical barrier to prevent the early embryo from interacting with the epithelium [9]. With so many unknown factors that regulate and maintain the normal tubal homeostatic environment, it is not surprising that progress in preventing the initiation and development of tubal EP in women has been so limited. Although it has been speculated that structural damage or functional disturbances (e.g., altered abnormal ciliary activity and/ or contractility) of the Fallopian tube may contribute actively to the development of EP $[2,10]$, the triggering molecular events are not to be delineated.

Several related risk factors have been proposed, such as pelvic infections, past or ever smoker and endometriosis [3,4]. Whether, and how, these risk factors directly induced local microenvironment changes and consequently triggered the tubal implantation needs to be explored. This question, which is difficult to address in humans, requires the development of adequate animal models. The function of the Fallopian tubes appears to involve orchestrated spatiotemporal alterations in transcriptome profiles. Therefore, extensive studies of temporal changes in the gene expression and in cell-specific signalling pathways in the Fallopian tube will be essential in order to understand the contribution of each of these risk factors to tubal function. Genetically-modified mouse models could help us to elucidate the underlying molecular mechanisms that are responsible for ciliary beating and muscular activity in the Fallopian tube in vivo under physiological conditions and in diseased states. An increased risk of tubal implantation caused by a delay in embryo transport from the Fallopian tube to the uterus, has been observed in new and existing studies using cannabinoid receptor (CB1)- [11], fatty acid amide hydrolase (FAAH)- [12], or Dicer1-knockout mice [13-15]. These mice exhibit tubal hypotrophy with the formation of prominent tubal cysts, leading to the disruption of tubal transport. The current results support the notion that the CB1-, FAAH-, and Dicer-mediated integrity of normal tubal structure and function is necessary for tubal transport. However, to date, there are no published reports regarding the expression and regulation of tubal CB1, FAAH, or Dicer1 in humans during tubal transport and in tubal EP. Using these knockout mouse models, the role of tubal CB1, FAAH, and Dicer1, and the underlying mechanisms of how aberrant these proteins lead to tube structural damage or functional disturbances can be investigated. These mouse models all have the potential to uncover the pathophysiological basis of tubal EP and allow researchers to design and test therapeutic targets. However, it is important to note that while mice are genetically similar to humans and both share features of tubal cell physiology, tubal EP is present in humans but is absent or rare in rodents $[2,10]$. Although genetically modified mice allow rigorous testing of mechanistic hypotheses, animal experiments do require judicious interpretation and recognition of their limitations.

We have assumed that both stimulatory and inhibitory cellular and paracrine mechanisms may contribute to the Fallopian tubal implantation (Figure 1). Each mechanism may contribute to tubal EP, as triggered by different pathological signals. Before we can address the pathophysiological basis of the tubal EP, however, we must elucidate the role of each of these mechanisms in the tubal EP. It may be reasonable to use mouse CB1-, FAAH, and Dicer1-knockout models to test each possible mechanism that has been proposed (Figure 1).

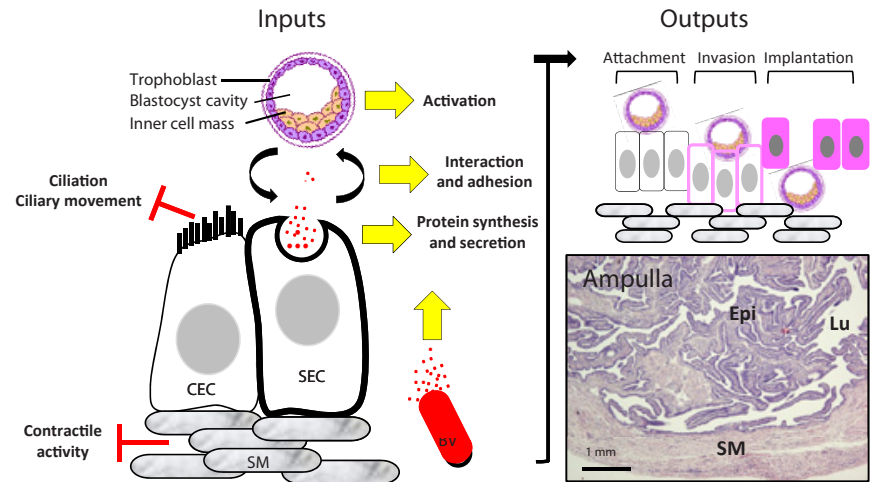

Figure 1: Proposed mechanisms of tubal ectopic pregnancy in humans. Bidirectional inputs—stimulation (yellow arrow) and inhibition (red bar)—may affect output responses of the Fallopian tube during early embryo transport Note that not all proposed cellular and paracrine processes are necessary for embryo implantation in the Fallopian tube under the same tubal environment. CEC, ciliated epithelial cells; SEC, secretory epithelial cells; SM, smooth muscle cells; BV, blood vessel; Lu, lumen; Epi, epithelium.

*Corresponding author: Ruijin Shao, MD, PhD, Associate Professor, Department of Physiology/Endocrinology, Institute of Neuroscience and Physiology, The Sahlgrenska Academy at University of Gothenburg, Gothenburg 40530, Sweden Tel: +46 31 7863408; Fax: +46 31 7863512; E-Mail: ruijin.shao@fysiologi.gu.se

Received March 06, 2012; Accepted March 08, 2012; Published March 10, 2012

Citation: Shao R (2012) Defining the Molecular Mechanisms for Tubal Ectopic Pregnancy Using Mouse Models. J Steroids Hormon Sci 3:e102. doi:10.4172/21577536.1000e102

Copyright: (C) 2012 Shao R. This is an open-access article distributed under the terms of the Creative Commons Attribution License, which permits unrestricted use, distribution, and reproduction in any medium, provided the original author and source are credited. 
Citation: Shao R (2012) Defining the Molecular Mechanisms for Tubal Ectopic Pregnancy Using Mouse Models. J Steroids Hormon Sci 3:e102. doi:10.4172/2157-7536.1000e102

Page 2 of 2

\section{References}

1. Khan KS, Wojdyla D, Say L, Gulmezoglu AM, Van Look PF (2006) WHO analysis of causes of maternal death: a systematic review. Lancet 367: 10661074.

2. Shaw JL, Dey SK, Critchley HO, Horne AW (2010) Current knowledge of the aetiology of human tubal ectopic pregnancy. Hum Reprod Update 16: 432-444.

3. Barnhart KT (2009) Clinical practice. Ectopic pregnancy. N Engl J Med 361 379-387.

4. Farquhar CM (2005) Ectopic pregnancy. Lancet 366: 583-591.

5. Jurkovic D, Wilkinson $H$ (2011) Diagnosis and management of ectopic pregnancy. BMJ 342: 3397.

6. Jansen RP (1984) Endocrine response in the fallopian tube. Endocr Rev 5: $525-551$

7. Shao R, Egecioglu E, Weijdegard B, Kopchick JJ, Fernandez-Rodriguez J, et al. (2007) Dynamic regulation of estrogen receptor-alpha isoform expression in the mouse fallopian tube: mechanistic insight into estrogen-dependent production and secretion of insulin-like growth factors. Am J Physiol Endocrino Metab 293: 1430-1442.

8. Shao R, Weijdegard B, Fernandez-Rodriguez J, Egecioglu E, Zhu C, et al. (2007) Ciliated epithelial-specific and regional-specific expression and regulation of the estrogen receptor-beta2 in the fallopian tubes of immature rats: a possible mechanism for estrogen-mediated transport process in vivo. Am J Physiol Endocrinol Metab 293: 147-158.

9. Hunter RH (2012) Components of oviduct physiology in eutherian mammals. Biol Rev Camb Philos Soc 87: 244-255.

10. Shao R (2010) Understanding the mechanisms of human tubal ectopic pregnancies: new evidence from knockout mouse models. Hum Reprod 25 584-587.

11. Wang H, Xie H, Dey SK (2008) Loss of cannabinoid receptor CB1 induces preterm birth. PLoS One 3: 3320 .

12. Wang $\mathrm{H}$, Xie H, Guo $\mathrm{Y}$, Zhang H, Takahashi T, et al. (2006) Fatty acid amide hydrolase deficiency limits early pregnancy events. J Clin Invest 116: 2122 2131.

13. Gonzalez G, Behringer RR (2009) Dicer is required for female reproductive tract development and fertility in the mouse. Mol Reprod Dev 76: 678-688.

14. Hong X, Luense LJ, McGinnis LK, Nothnick WB, Christenson LK (2008) Dicer1 is essential for female fertility and normal development of the female reproductive system. Endocrinology 149: 6207-6212.

15. Nagaraja AK, Andreu-Vieyra C, Franco HL, Ma L, Chen R, et al. (2008) Deletion of Dicer in somatic cells of the female reproductive tract causes sterility. Mol Endocrinol 22: 2336-2352. 